\title{
Análise das dificuldades de futuros pedagogos no processo de resolução de problemas geométricos ${ }^{1}$
}

\author{
Analysis of the difficulties of future educators in the process of solving geometric problems \\ Análisis de las dificultades de los futuros pedagogos en el proceso de resolución de \\ problemas geométricos
}

\author{
Érika Janine Maia-Afonso ${ }^{2}$ \\ Universidade Estadual do Paraná (UNESPAR), Apucarana, PR, Brasil \\ https://orcid.org/0000-0003-1850-3434, 9 http://lattes.cnpq.br/2252126588163533

\begin{abstract}
Marcelo Carlos de Proença ${ }^{3}$
Universidade Estadual de Maringá (UEM), Maringá, PR, Brasil
\end{abstract} \\ https://orcid.org/0000-0002-6496-4912, 9 http://lattes.cnpq.br/9198626057262085
}

\begin{abstract}
Resumo: Este estudo teve por objetivo analisar as dificuldades de futuros pedagogos no uso de seus conhecimentos para solucionar problemas que envolvem a geometria dos anos iniciais do Ensino Fundamental. Participaram da pesquisa 21 acadêmicos matriculados na disciplina de Metodologia do Ensino de Matemática II do curso de pedagogia de uma universidade pública localizada no noroeste do estado do Paraná. A coleta de dados se deu pela aplicação de uma prova escrita composta de cinco problemas geométricos. A análise das respostas dos participantes foi focada nas etapas de resolução de problemas: representação, planejamento, execução e monitoramento. Os resultados mostraram que os futuros pedagogos tiveram dificuldades para interpretar os enunciados, no que tange, sobretudo, à compreensão do termo planificação, ao reconhecimento da essência do problema e à identificação de informações irrelevantes. Além disso, apresentaram dificuldades nas etapas do planejamento e da execução de procedimentos e estratégias de resolução. A pesquisa evidenciou que as habilidades de orientação espacial, visualização e raciocínio espacial dos respectivos futuros pedagogos não foram bem desenvolvidas, o que implicou em dificuldades e limites para resolverem problemas geométricos que são destinados a alunos dos anos iniciais.
\end{abstract}

Palavras-chave: Geometria; conhecimento; formação inicial de professores; pensamento geométrico.

Abstract: This study aimed to analyze the difficulties of future educator in using their knowledge to solve problems involving the geometry of the Early Years of Elementary School. Twenty-one students enrolled in the discipline of Methodology of Teaching Mathematics II of the education of a public university located in the northwest of the state of Paraná participated in the research. Data collection was carried out by applying a written test composed of five geometric problems. The analysis of the participants' answers was focused on the problem solving steps: representation, planning, execution and monitoring. The results showed that future educator had difficulties in interpreting the statements, with regard, above all, to the understanding of the term planning, the recognition of the essence of the problem and the identification of irrelevant information. In addition, they presented difficulties in the stages of planning and executing procedures and resolution strategies. The research showed that the skills of spatial orientation, visualization and spatial reasoning of the respective future educator were not well developed, which implied difficulties and limits to solve geometric problems that are intended for students in the early years.

Keywords: Geometry; knowledge; initial teacher training; geometric thinking.

\footnotetext{
${ }^{1}$ Artigo apresentado no I Simpósio de Resolução de Problemas na Educação Matemática (I SiRPEM), da Universidade Estadual de Maringá, Maringá, Paraná, realizado nos dias 29 e 30 de julho de 2021.

${ }^{2}$ Currículo sucinto: Licenciada em Matemática pela Universidade Estadual de Maringá, doutora em Educação para a Ciência e a Matemática pela Universidade Estadual de Maringá. Professora colaboradora pela Universidade Estadual do Paraná, Campus Apucarana. Contribuição de autoria: Análise formal, conceituação, curadoria de dados, escrita - primeira redação, escrita - revisão e edição, investigação, metodologia, supervisão, validação e visualização. Contato: erikajaninemaia@gmail.com.

${ }_{3}^{3}$ Currículo sucinto: Licenciado em Matemática pela Universidade Estadual Paulista Júlio de Mesquita Filho (UNESP), Campus de Bauru, SP, doutor em Educação para a Ciência pela Universidade Estadual Paulista Júlio de Mesquita Filho. Professor pelo Departamento de Matemática da Universidade Estadual de Maringá. Contribuição de autoria: Análise formal, conceituação, curadoria de dados, escrita - primeira redação, escrita - revisão e edição, investigação, metodologia, supervisão, validação e visualização. Contato: mcproenca@uem.br.
} 
Resumen: Este estudio tuvo como objetivo analizar las dificultades de futuros pedagogos para utilizar sus conocimientos en la resolución de problemas relacionados con la geometría de los primeros años de la escuela primaria. Participaron de la investigación 21 estudiantes matriculados en la asignatura de Metodología de la Enseñanza de Matemáticas II del curso de Pedagogía de una universidad pública ubicada en el noroeste del estado de Paraná. La recolección de datos se realizó mediante la aplicación de una prueba escrita compuesta por cinco problemas geométricos. El análisis de las respuestas de los participantes se centró en los pasos de resolución de problemas: representación, planificación, ejecución y monitorización. Los resultados mostraron que los futuros pedagogos tenían dificultades para interpretar los enunciados, en lo que respecta, sobre todo, a la comprensión del término planificación, al reconocimiento de la esencia del problema y a la identificación de información irrelevante. Además, presentaron dificultades en las etapas de planificación y ejecución de procedimientos y estrategias de resolución. La investigación mostró que las habilidades de orientación espacial, visualización y razonamiento espacial de los respectivos Pedagogos en formación no estaban bien desarrolladas, lo que implicó dificultades y limitaciones para resolver problemas geométricos que se destinan a estudiantes de los primeros años de enseñanza primaria.

Palabras clave: Geometría; conocimiento; formación inicial de profesores; pensamiento geométrico.

Data de submissão: 23 de setembro de 2021.

Data de aprovação: 22 de outubro de 2021.

\section{Introdução}

Resolver problemas de matemática, segundo Echeverría (1998), é algo fundamental para que todo indivíduo possa desenvolver estratégias de raciocínio lógico e de pensamento aplicáveis em diferentes circunstâncias da vida cotidiana, bem como em diferentes áreas do conhecimento. A autora recomenda que a solução de problemas seja apresentada a crianças desde cedo, já nos primeiros anos da educação formal, com vistas a situações coerentes com o nível de conhecimento prévio desse aluno.

Os conteúdos matemáticos que integram o currículo dos anos iniciais do Ensino Fundamental são importantes para a resolução de problemas da vida comum, justamente pelo seu caráter prático e por favorecerem o desenvolvimento de capacidades intelectuais que ocupam um lugar de grande relevância na educação em geral, como é o caso da geometria. Segundo Fonseca et al. (2011), a geometria contribui para a ampliação e para a sistematização do conhecimento espontâneo adquirido por uma criança no espaço em que ela vive. Logo, é de fundamental importância que os professores que ministrarão aulas nos primeiros anos do ensino regular tenham conhecimentos geométricos bem formados em sua estrutura cognitiva, sabendo utilizá-los corretamente na resolução de problemas matemáticos.

Apesar do destaque dado à geometria e a sua relação com a resolução de problemas, em uma busca na literatura encontram-se pesquisas como as de Almeida (2009), Rosa (2009), Maia e Proença (2016) e Martins (2016), as quais mostram que acadêmicos concluintes de cursos de pedagogia possuem dificuldades para solucionar problemas matemáticos e para ensinar a matemática para alunos dos anos iniciais do Ensino Fundamental. Tais dificuldades são preocupantes, uma vez que esses futuros pedagogos poderão, em breve, assumir a 
responsabilidade de desenvolver as capacidades cognitivas desses alunos ao ministrarem aulas para esse nível de ensino.

Isso posto, pesquisas que se propõem a identificar os conhecimentos que futuros pedagogos mobilizam na resolução de problemas geométricos são pertinentes. Neste trabalho, objetiva-se analisar as dificuldades de futuros pedagogos no uso de seus conhecimentos para solucionarem problemas que envolvem a geometria dos anos iniciais do Ensino Fundamental. Com a análise das dificuldades observadas, pretende-se contribuir para a elaboração de novas pesquisas/perspectivas voltadas à formação de professores dos anos iniciais.

\section{Conhecimentos geométricos}

Rodrigues e Serrazina (2017), quanto ao Ensino Fundamental, afirmam que, nesse nível de aprendizado, o pensamento geométrico é mais centrado em imagens e em conceitos geométricos que não consideram as propriedades das figuras. Jones (2002), por sua vez, argumenta que, nessa etapa escolar, a geometria deve ser tida como um assunto que permite uma abordagem prática. Isso porque ela oferece ao professor a oportunidade de trabalhar com o aluno uma variedade de recursos exploratórios e investigativos das propriedades dos objetos e das formas geométricas, pensando sempre na solução de problemas característicos da vida comum.

Para Santos e Oliveira (2017), no campo da geometria, há dois importantes aspectos a serem desenvolvidos com alunos dos anos iniciais do Ensino Fundamental, quais sejam, as habilidades de visualização e de raciocínio espacial. Segundo Jones (2002, p. 125, tradução nossa), a habilidade de visualização "[...] permite aos alunos uma maneira de explorar matemática e outros problemas sem a necessidade de produzir diagramas precisos ou usar representações simbólicas". Já o raciocínio espacial foi definido por Clements e Battista (1992) como um conjunto de processos cognitivos cujas representações mentais dos objetos, relações e transformações espaciais são construídas e manipuladas. Para Jones (2002), o raciocínio espacial corresponde à formação de ideias mediante o estabelecimento de relações espaciais entre os objetos, constituindo um tipo de atividade mental que possibilita criar imagens espaciais e manipulá-las durante a resolução prática e teórica de problemas.

Além disso, outro ponto central da geometria é a orientação espacial, que está associada à capacidade humana de locomoção, movimentação e localização de pontos e de objetos no espaço. Conforme apontam Clements e Battista (1992), a orientação espacial envolve compreender e operar as relações entre as posições de objetos no espaço com a relação à sua própria posição.

Desse modo, o desenvolvimento de habilidades de visualização, de raciocínio e de orientação espacial são importantes não apenas para a compreensão da geometria em si, 
enquanto uma disciplina escolar, mas também para a construção de experiências baseadas em estímulos visuais presentes na realidade social do aluno, de maneira que ele possa interpretar e compreender a natureza de tais estímulos, e, ainda, aplicar seus conhecimentos na resolução dos problemas que a ele se apresentam (JONES, 2002).

\section{O processo de resolução de problemas}

No processo de resolução de problemas, deve-se destacar, inicialmente, que o sujeito se deparará com determinada situação matemática e deverá reconhecer essa situação como sendo um problema. Nesse sentido, entende-se que o problema é obtido "[...] quando o sujeito se depara com uma situação que o motiva a buscar uma resposta e reestruturar os elementos presentes na estrutura, de forma a chegar a um resultado" Brito (2010, p. 21). Conforme aponta Proença (2018),

[...] uma situação de Matemática se torna um problema quando a pessoa precisa mobilizar conceitos, princípios e procedimentos matemáticos aprendidos anteriormente para chegar a uma resposta. Não se trata, assim, do uso direto de uma fórmula ou regra conhecidas - quando isso ocorre, a situação tende a se configurar como um exercício. (PROENÇA, 2018, p. 17-18).

Em uma abordagem cognitiva da resolução de problemas, focada na teoria do processamento de informações, Brito (2010) analisa apontamentos de diversos autores e conclui que, de modo geral, todos utilizam os mesmos procedimentos para definir as fases/etapas do pensamento durante a solução de um problema matemático, a saber: 1) representação; 2) planejamento; 3) execução e 4) monitoramento. Brito (2010), no entanto, não define claramente cada uma dessas etapas no seu trabalho. Isso foi feito por Proença (2018), que, baseado em autores como Mayer (1992) e Krutetskii (1976), adotando as mesmas nomenclaturas propostas por Brito (2010), descreveu as quatro fases/etapas do raciocínio matemático, as quais serão apresentadas nos parágrafos seguintes.

A primeira etapa, concernente à representação, é o momento em que temos "[...] à compreensão ou à interpretação do problema pela pessoa que tenta solucioná-lo" (PROENÇA, 2018, p. 27), de modo que o solucionador constrói a representação mental do problema fazendo uso de seus conhecimentos linguísticos e semânticos, a fim de compreender a situação proposta. Segundo Proença (2018), no que tange aos conhecimentos linguísticos, o solucionador deverá

[...] realizar uma compreensão com base no que sua língua materna evidencia sobre a estrutura mais geral do problema (quem comete a ação, objetos envolvidos), isto é, reconhecer as palavras. $\mathrm{E}$, no caso do conhecimento semântico, a compreensão se dá, justamente com base no conhecimento da pessoa sobre os termos matemáticos que aparecem no problema e também sobre as relações que se estabelecem entre esses termos (PROENÇA, 2018, p. 27).

Para que o problema seja devidamente compreendido, o sujeito precisará, na etapa da representação, mobilizar conhecimentos matemáticos construídos ao longo do seu processo de escolarização, o que caracteriza o emprego do conhecimento esquemático. Para Proença (2018), 
esse conhecimento envolve o reconhecimento da essência do problema, o que consiste em identificar, por exemplo, se é um problema de álgebra, de geometria ou de aritmética, associando aos conceitos e procedimentos matemáticos aprendidos anteriormente, e que serão utilizados na resolução da situação matemática proposta. Desse modo, a representação do problema em si envolve conhecimentos linguísticos, semânticos e esquemáticos.

A segunda etapa, de planejamento, diz respeito ao momento em que o solucionador cria e elabora um plano para, a partir dele, tentar obter uma solução para a situação que tem diante de si. Assim, o solucionador organiza estrategicamente a informação buscando uma representação que o auxilie na execução da estratégia desenvolvida, algo que, segundo Proença (2018), demanda o uso do conhecimento estratégico.

Já na terceira etapa, de execução, com base em uma ação procedimental em cálculos, desenhos e outras formas de representações, o solucionador executa a estratégia previamente elaborada no planejamento. Proença (2018, p. 28) entende que o domínio do conhecimento procedimental do solucionador revelará "[...] a habilidade da pessoa para o uso de seu pensamento lógico no estabelecimento de relações quantitativas e espaciais". Por fim, a etapa do monitoramento corresponde ao ato de avaliar a solução obtida, quer dizer, examinar se ela é coerente com a situação proposta, para só então validar todo o processo de solução que foi construído.

Destaca-se que essas etapas não são seguidas de maneira linear pelo solucionador do problema, uma vez que isso depende do padrão de pensamento de cada pessoa. Sendo assim, o processo de construção da solução dependerá do quão bem formado esses conhecimentos estão em cada indivíduo. Logo, o êxito na resolução de um problema está diretamente relacionado aos conhecimentos que o solucionador tem armazenado na memória e à maneira como ele os mobilizará.

\section{Metodologia}

A pesquisa apresentada neste artigo possui características de uma abordagem qualitativa, ou seja, os dados são construídos pelo pesquisador e ele se coloca como o principal instrumento investigativo (ALVES-MAZZOTTI; GEWANDSZNAJDER, 2002). Além disso, destaca-se o fato de ela constituir uma investigação qualitativa descritiva, posto que, durante a coleta de dados, foram registradas informações por meio de palavras e figuras, a fim de representar fielmente o que ocorreu naquele ambiente. Os registros não foram reduzidos a números ou a símbolos numéricos apenas, pois foram considerados na sua totalidade, de modo a contemplar toda a sua riqueza (BOGDAN; BIKLEN, 1994). 
Os participantes foram 21 acadêmicos matriculados no primeiro semestre do ano de 2020 na disciplina de Metodologia do Ensino de Matemática II do curso de pedagogia de uma universidade pública localizada no noroeste do Paraná.

Para a coleta de dados, utilizou-se uma prova escrita com cinco situações matemáticas (às quais iremos nos referir como $\mathrm{S} 1$, evoluindo até $\mathrm{S} 5$, respectivamente) envolvendo a geometria ensinada nos anos iniciais do Ensino Fundamental. Tal prova foi elaborada com base nos descritores da Prova Brasil, que indica as habilidades ou as competências em geometria que alunos do ensino regular devem adquirir no decorrer do Ensino Fundamental. Sendo assim, os descritores que serviram de referência para a elaboração da referida prova foram aqueles relacionados a "espaço e forma" e a "grandezas e medidas", a saber: D1. Identificar a localização e movimentação de objeto em mapas, croquis e outras representações gráficas; D2. Identificar propriedades comuns e diferenças entre poliedros e corpos redondos, relacionando figuras tridimensionais com suas planificações; D11. Resolver problema envolvendo o cálculo do perímetro de figuras planas, desenhadas em malhas quadriculadas; D12. Resolver problema envolvendo o cálculo ou estimativa de áreas de figuras planas, desenhadas em malhas quadriculadas.

As situações S1 e S3 foram elaboradas pelos pesquisadores, a S2 foi adaptada de Barbosa (2011), a S4 foi retirada na íntegra de Dante (2017) e a S5 foi reelaborada a partir de uma situação também proposta por Dante (2017). Levou-se em consideração para a (re)elaboração/seleção das questões, as habilidades e objetos de conhecimento que são apresentadas na Base Nacional Comum Curricular (BNCC) (BRASIL, 2018), documento que descreve os conteúdos geométricos a serem ministrados nos anos iniciais do Ensino Fundamental, segundo a série de estudo do aluno. No Quadro 1, portanto, as questões foram classificadas conforme a sua correspondência com os descritores de referência da Prova Brasil, incluindo as habilidades $^{1}$ e o ano escolar (em negrito na coluna BNCC) que a questão deveria/poderia ser trabalhada em sala de aula com o aluno do Ensino Fundamental, de acordo com a BNCC (BRASIL, 2018).

$\mathrm{Na}$ análise dos dados da prova de geometria, foram utilizados aspectos teóricos da resolução de problemas, para que fosse possível encontrar categorias e classificar as soluções apresentadas pelos futuros pedagogos, segundo as dificuldades tidas em cada uma das quatro etapas da resolução de problemas discutidas por Proença (2018): representação, planejamento, execução e monitoramento. Assim, quando o solucionador não apresentou dificuldade no desenvolvimento dessas quatro etapas, sua resposta foi considerada correta. Por outro lado, nos casos em que o solucionador apresentou dificuldades na etapa da representação, sua resposta foi

\footnotetext{
1 Descrever e representar a localização de pessoas e de objetos no espaço; reconhecer, nomear, associar e comparar figuras geométricas espaciais; medir, comparar e estimar área e perímetro de figuras planas. Para a descrição completa de cada item, consultar o link: http://basenacionalcomum.mec.gov.br/images/BNCC EI EF 110518 versaofinal site.pdf. Acesso em: 27 jun. 2021. 
considerada incorreta, isso porque, conforme aponta Sternberg (2000), uma representação inadequada do problema pode gerar um obstáculo à sua resolução, comprometendo todo o processo que será realizado a seguir, resultando em maiores dificuldades. Quando o solucionador apresentou dificuldades em ao menos uma das etapas, seja na de planejamento, de execução ou de monitoramento, sua resposta foi considerada parcialmente correta. Por fim, nos casos em que a situação ficou em branco, isto é, não resolvida, ela foi classificada como não fez.

Quadro 1 - Classificação das questões da prova de geometria de acordo com os descritores da Prova Brasil e as habilidades da BNCC

\begin{tabular}{|c|c|c|}
\hline Situação & Prova Brasil & BNCC \\
\hline S1 & D1 & EF01MA11; EF01MA12; EF02MA12; EF03MA12; EF04MA16 \\
\hline S2 & D2 & EF01MA13; EF02MA14; EF03MA13; EF04MA17; EF05MA16 \\
\hline S3 & D1 & EF01MA11; EF01MA12; EF02MA12; EF03MA12; EF04MA16 \\
\hline S4 & D11 & EF04MA20 \\
\hline S5 & D12 & EF04MA21 \\
\hline
\end{tabular}

Fonte: Elaborado pelos autores.

Deve-se salientar que, nessa prova, a análise do desempenho dos acadêmicos não foi feita somente com base nas respostas finais apresentadas por eles. Avaliou-se, sobretudo, a maneira como conceberam a resolução de problemas, as estratégias que utilizaram, bem como as etapas que adotaram no processo de solução. Os quadros e as tabelas têm o intuito de facilitar a visualização dos resultados obtidos e servem para exemplificar as dificuldades dos futuros pedagogos na resolução de problemas geométricos. Foram utilizados, também, palavras e figuras presentes nas respostas dos participantes, uma vez que, conforme indicam Bogdan e Biklen (1994), quando os dados recolhidos são em formas de palavras ou imagens, é papel do pesquisador analisar esses dados em toda a sua riqueza, respeitando sempre a maneira como foram registrados pelos participantes.

\section{Resultados e discussão}

Para um panorama geral das soluções dos 21 futuros pedagogos (representados pelas abreviações que vão de P1 a P21), tem-se, na Tabela 1, a classificação de cada situação, de acordo com o tipo de resposta de cada participante.

Tabela 1 - Tipos de respostas apresentadas pelos futuros pedagogos na prova de geometria

\begin{tabular}{|c|c|c|c|c|}
\hline Situação & Tipo de Resposta & Participante & TOTAL & (\%) \\
\hline \multirow{4}{*}{ S1 } & Não fez & P2 & 1 & 4,76 \\
\cline { 2 - 5 } & Correta & P20 & 1 & 4,76 \\
\cline { 2 - 6 } & $\begin{array}{c}\text { Parcialmente } \\
\text { correta }\end{array}$ & P4, P5, P6, P11, P13 & 5 & 23,81 \\
\hline
\end{tabular}




\begin{tabular}{|c|c|c|c|c|}
\hline & Incorreta & $\begin{array}{c}\mathrm{P} 1, \mathrm{P} 3, \mathrm{P} 7, \mathrm{P} 8, \mathrm{P} 9, \mathrm{P} 10, \mathrm{P} 12, \mathrm{P} 14, \mathrm{P} 15, \mathrm{P} 16, \mathrm{P} 17, \mathrm{P} 18, \\
\mathrm{P} 19, \mathrm{P} 21\end{array}$ & 14 & 66,67 \\
\hline \multirow{4}{*}{ S2 } & Não fez & P2 & 1 & 4,76 \\
\hline & Correta & $\mathrm{P} 4, \mathrm{P} 10, \mathrm{P} 12, \mathrm{P} 17, \mathrm{P} 18, \mathrm{P} 19$ & 6 & 28,57 \\
\hline & $\begin{array}{l}\text { Parcialmente } \\
\text { correta }\end{array}$ & P5, P6, P20 & 3 & 14,29 \\
\hline & Incorreta & $\mathrm{P} 1, \mathrm{P} 3, \mathrm{P} 7, \mathrm{P} 8, \mathrm{P} 9, \mathrm{P} 11, \mathrm{P} 13, \mathrm{P} 14, \mathrm{P} 15, \mathrm{P} 16, \mathrm{P} 21$ & 11 & 52,38 \\
\hline \multirow{4}{*}{ S3 } & Não fez & P2 & 1 & 4,76 \\
\hline & Correta & $\mathrm{P} 1, \mathrm{P} 5, \mathrm{P} 7, \mathrm{P} 16, \mathrm{P} 18, \mathrm{P} 19, \mathrm{P} 20$ & 7 & 33,33 \\
\hline & $\begin{array}{l}\text { Parcialmente } \\
\text { correta }\end{array}$ & $\begin{array}{l}\mathrm{P} 3, \mathrm{P} 4, \mathrm{P} 6, \mathrm{P} 8, \mathrm{P} 9, \mathrm{P} 10, \mathrm{P} 11, \mathrm{P} 12, \mathrm{P} 13, \mathrm{P} 14, \mathrm{P} 15, \mathrm{P} 17, \\
\mathrm{P} 21\end{array}$ & 13 & 61,90 \\
\hline & Incorreta & - & 0 & 0 \\
\hline \multirow{4}{*}{ S4 } & Não fez & P2, P9, P13 & 3 & 14,29 \\
\hline & Correta & $\mathrm{P} 1, \mathrm{P} 3, \mathrm{P} 11, \mathrm{P} 12, \mathrm{P} 14, \mathrm{P} 15, \mathrm{P} 17, \mathrm{P} 18, \mathrm{P} 19, \mathrm{P} 20$ & 10 & 47,62 \\
\hline & $\begin{array}{l}\text { Parcialmente } \\
\text { correta }\end{array}$ & P4, P6, P10, P16, P21 & 5 & 23,81 \\
\hline & Incorreta & P5, P7, P8 & 3 & 14,29 \\
\hline \multirow{4}{*}{ S5 } & Não fez & $\mathrm{P} 2, \mathrm{P} 9, \mathrm{P} 13$ & 3 & 14,29 \\
\hline & Correta & $\mathrm{P} 1, \mathrm{P} 12, \mathrm{P} 15, \mathrm{P} 18, \mathrm{P} 21$ & 5 & 23,81 \\
\hline & $\begin{array}{l}\text { Parcialmente } \\
\text { correta }\end{array}$ & P3, P5, P6, P7, P8, P16, P20 & 7 & 33,33 \\
\hline & Incorreta & $\mathrm{P} 4, \mathrm{P} 10, \mathrm{P} 11, \mathrm{P} 14, \mathrm{P} 17, \mathrm{P} 19$ & 6 & 28,57 \\
\hline
\end{tabular}

Fonte: Elaborado pelos autores.

$\mathrm{Na}$ análise da Tabela 1, verifica-se que o agrupamento das categorias "parcialmente correta" e "incorreta", nas cinco situações, corresponde à maioria das respostas, o que mostra que os futuros pedagogos possuem dificuldades para interpretar e solucionar problemas no que tange às etapas de resolução de problemas (representação, planejamento, execução e monitoramento). Sendo assim, devido aos resultados evidenciados na Tabela 1, na busca de identificar quais são as dificuldades apresentadas pelos respondentes em cada uma das situações, foi elaborado o Quadro 2, o qual especifica as dificuldades tidas pelos acadêmicos em cada uma das quatro etapas aqui descritas.

Quadro 2 - Etapa que o participante apresentou dificuldade por situação da prova de geometria

\begin{tabular}{|c|c|c|}
\hline Situação & Etapa & Participante \\
\hline \multirow[t]{2}{*}{ S1 } & Representação & $\begin{array}{c}\mathrm{P} 1, \mathrm{P} 3, \mathrm{P} 4, \mathrm{P} 7, \mathrm{P} 8, \mathrm{P} 9, \mathrm{P} 10, \mathrm{P} 12, \mathrm{P} 14, \mathrm{P} 15, \mathrm{P} 16, \mathrm{P} 17, \mathrm{P} 18, \mathrm{P} 19, \\
\text {, }\end{array}$ \\
\hline & Planejamento & $\mathrm{P} 7, \mathrm{P} 6, \mathrm{P} 11, \mathrm{P} 13$ \\
\hline \multirow{2}{*}{ S2 } & Representação & $\mathrm{P} 1, \mathrm{P} 3, \mathrm{P} 7, \mathrm{P} 8, \mathrm{P} 9, \mathrm{P} 11, \mathrm{P} 13, \mathrm{P} 14, \mathrm{P} 15, \mathrm{P} 16, \mathrm{P} 21$ \\
\hline & Execução & P5, P6, P20 \\
\hline \multirow{2}{*}{ S3 } & Planejamento & $\mathrm{P} 3, \mathrm{P} 6, \mathrm{P} 10, \mathrm{P} 11, \mathrm{P} 12, \mathrm{P} 13, \mathrm{P} 14, \mathrm{P} 15$ \\
\hline & Execução & $\mathrm{P} 4, \mathrm{P} 8, \mathrm{P} 9, \mathrm{P} 17, \mathrm{P} 21$ \\
\hline \multirow{2}{*}{ S4 } & Representação & $\mathrm{P} 5, \mathrm{P} 7, \mathrm{P} 8$ \\
\hline & Execução & $\mathrm{P} 4, \mathrm{P} 6, \mathrm{P} 16$ \\
\hline
\end{tabular}




\begin{tabular}{|c|c|c|}
\hline & Monitoramento & P10, P21 \\
\hline \multirow{2}{*}{ S5 } & Planejamento & P16, P20 \\
\cline { 2 - 3 } & Monitoramento & P3, P5, P6, P7, P8 \\
\hline
\end{tabular}

Fonte: Elaborado pelos autores.

No Quadro 2, verifica-se que, em três das cinco situações, houve dificuldades relacionadas à etapa de representação do problema, o que indica dificuldade na compreensão da situação proposta. Resultados semelhantes foram encontrados nas pesquisas de Almeida (2009) e nos estudos de Maia e Proença (2016). De modo específico, Maia e Proença (2016) relatam que, após a solução de dez situações de geometria por futuros pedagogos, a maior dificuldade notada foi relacionada à etapa da representação, correspondendo a 54,67\% $(n=42)$. Ou seja, nesse caso, a maioria dos acadêmicos demonstrou ter alguma dificuldade para compreender, interpretar e representar os problemas propostos. Nessa mesma perspectiva, Almeida (2009, p. 152) mostra que futuros professores apresentam dificuldades para compreender problemas simples e "[...] não costumam usar estratégias exploratórias para resolver problemas".

Com o intuito de evidenciar os conhecimentos (linguístico, semântico, esquemático, estratégico e procedimental) mobilizados por futuros pedagogos nas quatro etapas de resolução de problemas (PROENÇA, 2018), a seguir, é descrito o processo de resolução de cada situação. Para isso, as situações foram agrupadas em uma sequência que considera a interseção de conteúdos que as envolvem, na ordem S1 e S3, S2, S4 e S5.

O enunciado da S1 pode ser observado na Figura 1.

Figura 1 - S1 da prova de geometria

\begin{tabular}{|c|c|c|c|c|c|}
\hline \multicolumn{6}{|c|}{$\begin{array}{l}\text { 1) A professora de Matemática de Fernando passou um trabalho que ele deverá fazer em dupla } \\
\text { com sua colega Miranda. Para isso, ele precisará sair de sua casa (número 1) e ir até a casa da } \\
\text { sua colega (número } 7 \text { ), que estão representadas pela malha quadriculada abaixo. }\end{array}$} \\
\hline & & - & & & $R=A$ \\
\hline & - & 10 & 11 & $x_{1}$ & $\operatorname{sen} B$ \\
\hline & & & & & Row $C$ \\
\hline & & & 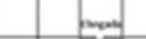 & & Kma D \\
\hline & 5 & - & , & $\cdot$ & $\operatorname{Rog} E$ \\
\hline & & & & & $\operatorname{Ran} \mathrm{F}$ \\
\hline & & & & & Ras C \\
\hline$=$ & 11 & 2 & 3 & 4 & $\mathrm{Rm} H$ \\
\hline & & & & & Rus 1 \\
\hline \multicolumn{6}{|c|}{$\begin{array}{l}\text { Porém ao sair de casa, Fernando lembrou que primeiro deveria passar na escola (número 10) pegar } \\
\text { seu material e depois seguir para casa de Miranda. Sabendo que cada quadradinho da figura acima } \\
\text { corresponde a uma quadra das ruas do bairro e que as portas de acesso para os estabelecimentos } \\
\text { estão localizadas exatamente no meio da quadra, represente na malha quadriculada qual poderá } \\
\text { ser o trajeto percorrido por Fernando até chegar à casa de Miranda e, em seguida, descreva esse } \\
\text { percurso nas linhas abaixo. }\end{array}$} \\
\hline
\end{tabular}

Fonte: Elaborado pelos autores - Prova de geometria.

A principal dificuldade encontrada por 15 participantes na resolução da $\mathrm{S} 1$ teve relação com a etapa de representação do problema. Nota-se que os acadêmicos não atentaram para a 
seguinte informação constante no enunciado: "as portas de acesso para os estabelecimentos estão localizadas exatamente no meio da quadra". Na Figura 2, vê-se a solução ${ }^{2}$ apresentada pelo P13. Ao indicar, na malha quadriculada, o caminho a ser percorrido por Fernando, o P13 pintou o quadradinho inteiro, desconsiderando a porta de acesso para a saída de sua casa, para a entrada na escola e para a chegada na casa de Miranda, todas localizadas no meio da quadra.

Figura 2 - Solução da S1 do P13

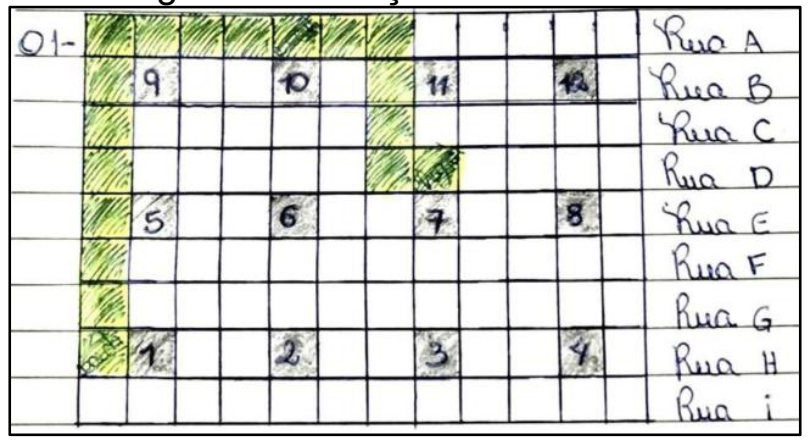

Fonte: Prova de geometria do P13.

Tal dificuldade na interpretação relaciona-se com o conhecimento esquemático conforme apontado por Proença (2018). Na leitura do enunciado da S1, os solucionadores não consideraram um dado caracterizado como relevante para a resolução do problema, a fim de que pudessem planejar uma solução adequada. Já no que tange às dificuldades dos participantes na etapa de planejamento, na $\mathrm{S} 1$, percebe-se que os futuros pedagogos não adotaram um referencial na hora de dar a sua resposta, apresentando dificuldades no conhecimento estratégico, como pode ser observado na solução do $\mathrm{P} 17$, o qual escreveu o seguinte: "Fernando saiu da rua $\mathrm{H} \mathrm{e}$ andou 12 quadras até a escola A. Após, andou 7 quadras até a Rua D".

Verifica-se que a resposta do P17 não indica, detalhadamente, qual o caminho a ser percorrido por Fernando, apenas apresenta quantas quadras deverão ser percorridas, sem dar informações de lateralidade: virar à direita ou à esquerda; andar pela rua $A$ durante todo percurso ou passar pelas Ruas B e C. A descrição minuciosa do percurso é importante, pois, como apontaram Fonseca et al. (2011), contribui para a ampliação e para a sistematização do conhecimento que a criança tem do espaço em que vive. Enquanto futuros professores, os participantes deveriam apresentar descrições mais pormenorizadas, para que os alunos tenham contato com resoluções completas.

As resoluções dos futuros pedagogos para a $S 3$, que tem seu enunciado reproduzido na Figura 3, mostrou que, nessa situação, as dificuldades encontradas tiveram relação com as etapas de planejamento e de execução.

\footnotetext{
2 Uma possível resposta correta seria: Fernando sai de sua casa, na rua $\mathrm{H}$, vira à direita, sobe em direção à rua $\mathrm{A}$ e anda seis quadras e meia. Ao chegar na rua A, vira novamente à direita, horizontalmente, andando três quadras e meia até a escola. Ao sair da escola, anda duas quadras e meia horizontalmente para à direita, desce três quadras verticalmente e anda meia quadra para a sua esquerda (movimento horizontal à direita no mapa), chegando na casa de sua colega Miranda.
} 
Figura 3 - S3 da prova de geometria

3) (Barbosa, 2011, p. 178) O professor de Alice propôs uma brincadeira à sua turma: "Boneco obediente $^{n}$. Nesta brincadeira os jogadores precisam traçar um caminho a partir de um comando que indica se é preciso ir para frente, para a direita, para a esquerda ou para trás. A figura abaixo representa os comandos dados pelo professor à Alice.

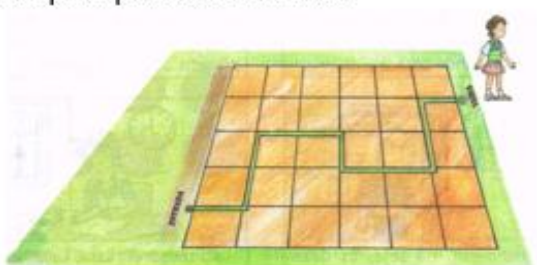

Quais os comandos o professor deu para Alice fazer esse percurso, considerando que cada lado do quadriculado é um passo?

Fonte: Elaborado pelos autores - Prova de geometria.

No que se refere ao planejamento, oito participantes demonstraram compreender o que deveria ser feito, porém, assim como já foi destacado em S1, não adotaram um referencial da posição de Alice. Esse fato, constatado tanto em S1 quanto em S3, revela que um dos temas centrais da geometria, que é a orientação espacial, conforme apontaram Clements e Battista (1992), não foi bem desenvolvido pelos futuros pedagogos, uma vez que eles, na sua maioria, demonstraram incapacidade de se locomover/movimentar no espaço tendo por base um ponto de referência.

No que tange à etapa de execução, observa-se que, ao descrever os comandos de movimentação, cinco participantes confundiram as noções de direita e esquerda em uma de suas passagens, revelando dificuldades referente ao conhecimento procedimental proposto por Proença (2018). Na visão de Clements e Battista (1992), a confusão das noções de direita e esquerda está justamente ligada à dificuldade de orientação espacial, o que, segundo Menezes et al. (2014), pode ser motivo de insucesso escolar. Os conhecimentos investigados na pesquisa, conforme já sublinhado, são aqueles que se espera que os alunos dos anos iniciais adquiram.

$\mathrm{Na}$ situação S2, cujo enunciado consta na Figura 4, as dificuldades dos futuros pedagogos foram nas etapas de representação e de execução do problema.

De modo específico, na etapa da representação, observam-se dificuldades no conhecimento esquemático, pois os futuros professores demonstraram não compreender o que é planificação em relação ao seu conceito, como apontou P18: "Não me recordo os conceitos de planificação". Situações geométricas que envolvem a planificação contribuem para o desenvolvimento e para o aprimoramento da habilidade de visualização e de raciocínio espacial, habilidade essa que foi apontada por Santos e Oliveira (2017) como central no processo de ensino-aprendizagem da geometria e que futuros pedagogos envolvidos nesta pesquisa demonstraram não saber trabalhar com facilidade. 
Figura 4 - S2 da prova de geometria

2) Na Páscoa deste ano, Joana presenteou 5 sobrinhos com uma caixa de chocolates igual a que está representada na figura a seguir.

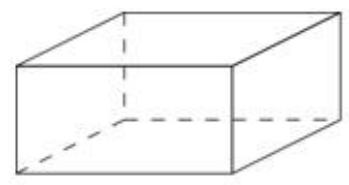

Para embrulhar todas as caixas que serão entregues, Joana foi até uma livraria comprar papel de presente e levou consigo $R \$ 20,00$, sendo duas notas de $R \$ 5,00$ e uma nota de $R \$ 10,00$. No momento da compra, a atendente lhe informou que para saber a quantidade de papel exata que será utilizada para embrulhar os presentes, é preciso conhecer a planificação da caixa de chocolates. Ajude Joana e faça a representação da planificação desta embalagem no espaço a seguir.

Essa planificação corresponde a qual sólido geométrico?

Fonte: Adaptado de Barbosa (2011) - Prova de geometria.

Além disso, as dificuldades no conhecimento esquemático podem, também, ser relacionadas ao fato de os futuros pedagogos terem utilizado informações supérfluas (excesso de dados) do enunciado da situação, uma vez que a S2 solicitava a planificação do sólido, ou seja, não há menção aos valores numéricos na questão da situação. Pode-se observar, na Figura 5, a solução do P21.

Figura 5 - Solução da S2 do P21

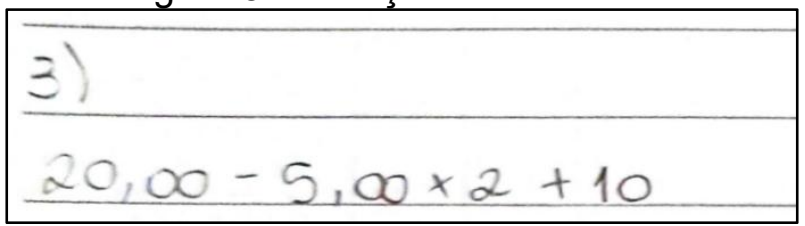

Fonte: Prova de geometria do P21.

Desse modo, P21 demonstrou não saber identificar e descartar as informações irrelevantes do enunciado da situação, uma habilidade necessária e que deve ser adquirida pelo solucionador, conforme apontou Proença (2018). Em se tratando da etapa da execução, foram classificados os participantes que realizaram planificações de maneira equivocada, como se pode ver na Figura 6 .

Figura 6 - Solução da S2 do P20

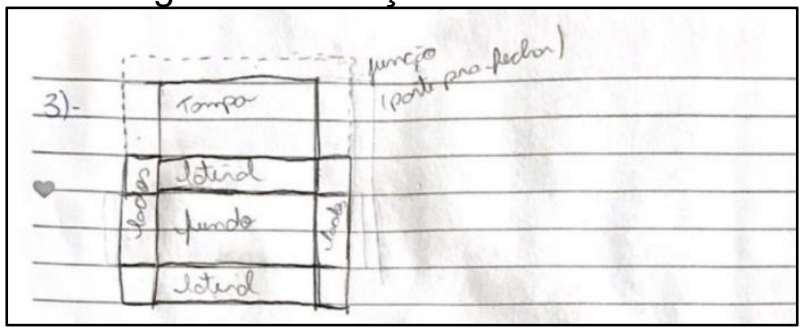

Fonte: Prova de geometria do P20.

Observa-se, na resolução de P20, uma compreensão do conceito de planificação; porém, no momento de realizar a planificação, o acadêmico não a fez de modo correto, o que é sinal de 
dificuldade de visualização e de raciocínio espacial. Uma possível solução adequada para essa situação seria a representada pela Figura 7 .

Figura 7 - Solução adequada para S2

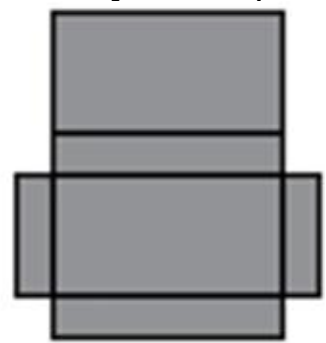

Fonte: Elaborado pelos autores.

Desse modo, acredita-se que associar a resolução dessa situação ao uso de um material que o aluno possa manipular, como a própria caixa de bombom, conforme apontou Jones (2002), pode ajudá-lo a superar dificuldades de resolução como as apresentadas. Sendo assim, as dificuldades vistas em S2 mostram que os participantes desta pesquisa não assimilaram devidamente os conceitos de planificação de um sólido geométrico, tampouco as relações entre figuras bidimensionais e tridimensionais. Resultado semelhante foi encontrado na pesquisa de Menezes et al. (2014), autores que aplicaram um teste contendo 21 questões de múltipla escolha para estudantes de três escolas superiores de Portugal. Os estudantes portugueses, naquele caso, apresentaram um percentual de acerto baixo no atinente a questões de raciocínio espacial e planificação do cubo, incluindo as propriedades de figuras 2D.

O enunciado da situação S4 foi representado na Figura 8.

Figura 8 - S4 da prova de geometria

4) Seu José preparou 2 canteiros para plantar verduras. Um deles tem a forma quadrada e o outro a forma retangular. Veja suas medidas, indicadas na figura.

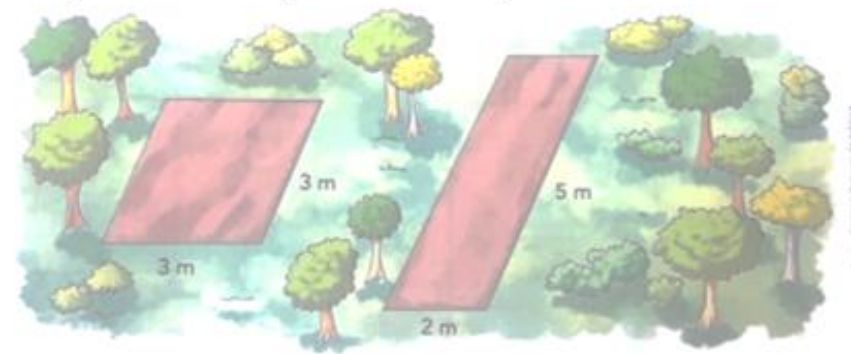

Fonte: Dante, Luiz Roberto. Projeto Ápis Matemática: $4^{\circ}$ ano, Editora ática, p. 62, 3. ed. São Paulo, 2017.

Cada lado dos quadradinhos da malha quadriculada abaixo representa 1 metro. Sabendo disso, desenhe os 2 canteiros de Seu José vistos de cima.

Quantos metros seu José percorrerá para caminhar pelo contorno do canteiro que tem a forma quadrada? E quantos metros percorrerá ao caminhar pelo canteiro de forma retangular?

Fonte: Dante (2017) - Prova de geometria. 
Em S4, as dificuldades dos acadêmicos na etapa da representação se relacionam ao fato de terem realizado o cálculo de área quando o correto seria o cálculo do perímetro, conforme a resolução de P8, que pode ser vista na Figura 9.

Figura 9 - Solução do P8 para S4

1) a) o canteiro que tem a forma quadrada jose tera que percorrer $3 \times 3=9$ metros

Fonte: Prova de geometria do P8.

Esse equívoco entre o conceito de área e de perímetro, também foi evidenciado na pesquisa de Pessoa (2010), que buscou identificar os procedimentos mobilizados por alunos do $6^{\circ}$ ano do Ensino Fundamental na resolução de atividades de cálculo de área de figuras planas com o auxílio da malha quadriculada. Como resultado, o referido autor aponta que os alunos não souberam diferenciar corretamente área e perímetro. Quando foi solicitado o cálculo da área de figuras planas, eles determinaram o perímetro.

Outra dificuldade dos futuros pedagogos aqui estudados relativas à visualização está no modo como eles fizeram a representação na malha quadriculada da vista frontal do canteiro. $O$ correto seria o desenho da vista superior (Figura 10).

Figura 10 - Solução do P7 para S4

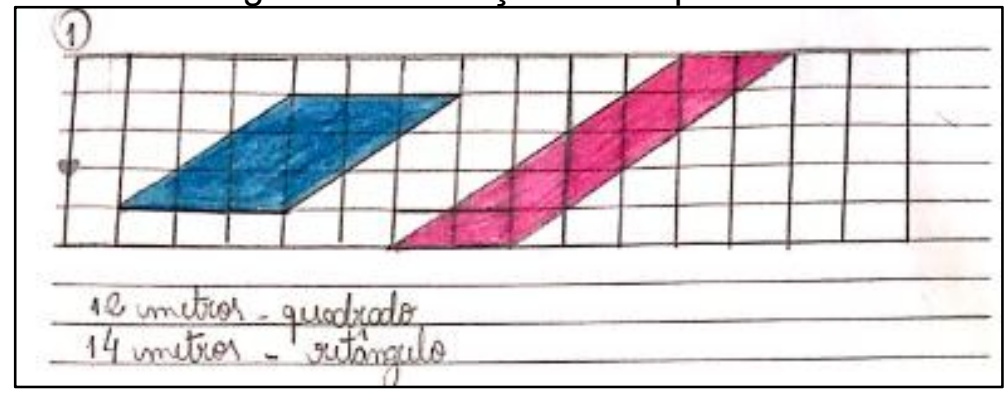

Fonte: Prova de geometria do P7.

Tais dificuldades evidenciadas na resolução da $S 4$, no concernente à etapa de representação do problema, têm relação com o conhecimento esquemático do sujeito. Neste estudo, os solucionadores não reconheceram corretamente a essência do problema e não identificaram os conceitos e os procedimentos matemáticos aprendidos anteriormente, os quais eram necessários para a resolução da situação apresentada, conforme também apontado por Proença (2018).

Vale ressaltar que, na resolução da S4, seria possível fazer o desenho, de acordo com a Figura 10. No entanto, isso foi um problema quando o quadriculado já tinha uma dimensão previamente estabelecida, de modo que o desenho não representaria o tamanho correto daquilo que é dado pela situação. Em suma, a resposta escrita por P7 está correta, mas o desenho elaborado por ele não é condizente com o que foi pedido no enunciado da situação. 
Outra etapa em que houve dificuldades na resolução da S4 foi a de execução. Na Figura 11 , representativa da resolução de $\mathrm{P} 16$, tem-se um exemplo dessa dificuldade.

Figura 11 - Solução do P16 para S4

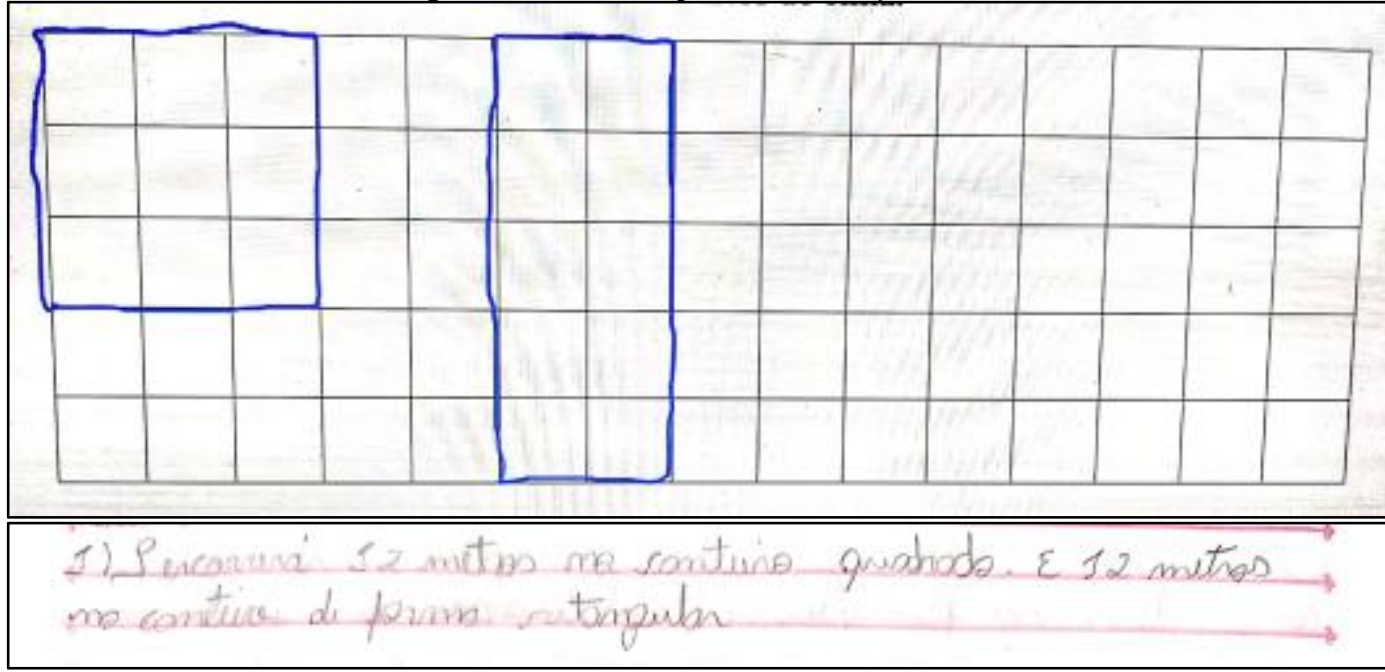

Fonte: Prova de geometria do P16.

Nota-se, na resposta de $\mathrm{P} 16$, que o contorno do perímetro realizado na malha quadriculada está correto; entretanto, no momento de apresentação da solução, o participante cometeu um erro procedimental ao realizar a contagem dos quadradinhos que compõem o contorno do canteiro retangular.

No que se refere à etapa de monitoramento, vê-se, na Figura 12, na resolução do P21, que, ao fornecer uma solução, o solucionador indicou a soma e a representação na malha quadriculada corretamente, mas, ao final, ao colocar os números 12 e 14, não os incluiu no contexto gerado pelo enunciado da situação, deixando de assinalar as unidades de medidas a serem consideradas.

Figura 12 - Solução do P21 para S4

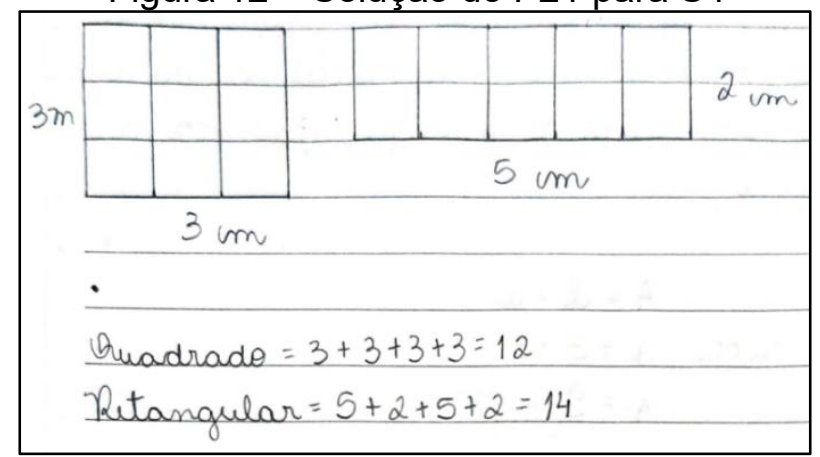

Fonte: Prova de geometria do P21.

A resposta fornecida por P21 (Figura 12) é preocupante, uma vez que ele pode ter a mesma atitude em sala de aula, e esse tipo de erro pode ser reproduzido por seus alunos. Essa resposta apresenta uma característica procedimental de não atenção à necessidade de relacionar a atividade solucionada com o contexto do qual ela faz parte. Essa habilidade é necessária e 
importante para o solucionador que lida com atividades de resolução de problemas, conforme apontado por Proença (2018).

A Figura 13 apresenta o enunciado da S5.

Figura 13 - S5 da prova de geometria

5) Lucca deseja construir uma horta no quintal de sua casa para cultivar legumes e hortaliças e para isso vai investir um total de $\mathrm{R} \$ 500,00$. Inicialmente, Lucca decidiu comprar sementes e adubo na venda do seu João que aproveitou a oportunidade para alertá-lo que antes de adubar a terra e plantar as sementes será preciso fazer a limpeza do local em que a horta será cultivada. A região triangular representada na figura a seguir, corresponde a parte do quintal em que Lucca construirá sua horta com suas respectivas medidas.

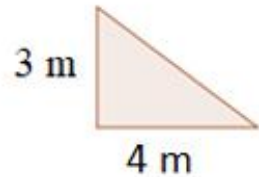

Sabendo que Lucca já gastou um total de $\mathrm{R} \$ 120,00$ do seu orçamento inicial na venda do seu João, qual será o valor correspondente a área da região que ele deverá limpar para cultivar sua horta?

\section{Fonte: Adaptado de Dante (2017) - Prova de geometria.}

Nessa situação, na etapa de planejamento, a dificuldade de P16 e P20 aparece associada ao uso de uma fórmula equivocada para encontrar a solução, conforme ilustra a Figura 14.

Figura 14 - Solução do P20 para a S5

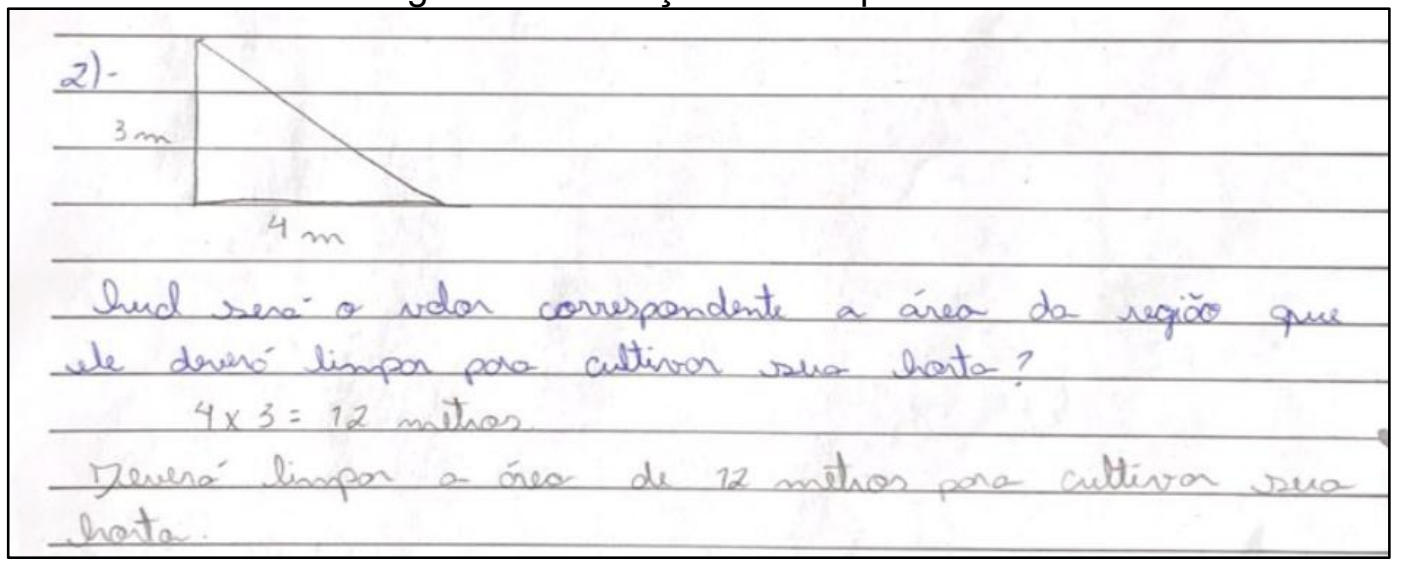

Fonte: Prova de geometria do P20.

Nessa solução, o participante reconheceu que a situação se referia ao conteúdo de área, porém, no momento de efetuar o cálculo, ele utilizou a multiplicação entre os dois lados do triângulo, o que lhe forneceria, na realidade, a área do retângulo. Assim, o participante revela ter feito uma extensão inadequada das fórmulas. Fato semelhante foi apontado por Douady e PerrinGlorian (1989), pesquisadoras francesas que buscaram compreender como alunos dos anos iniciais do ensino regular resolvem situações envolvendo o conceito de área de figuras planas. Elas mostram que uma das dificuldades deles foi a extensão inadequada de fórmulas, como tentar encontrar a área de um paralelogramo mediante o produto das duas dimensões, por exemplo. 
No que se refere à etapa do monitoramento, a Figura 15 apresenta a solução do P7, exemplificando outro tipo de dificuldade encontrada. Observa-se, em se tratando de um cálculo de área, a unidade de medida apresentada ao final deveria ser metro ao quadrado $\left(\mathrm{m}^{2}\right)$ e não metro, conforme colocado por P7.

Figura 15 - Solução do P7 para a S5

(2) $\frac{b . h}{2} \Rightarrow \frac{4.3}{2} \Rightarrow \frac{12}{2} \Rightarrow 6$ metros

Fonte: Prova de geometria do P7.

De modo geral, após a análise das situações S4 e S5, verificou-se que os futuros pedagogos tiveram dificuldade para solucionar questões relacionadas ao cálculo de área e de perímetro mediante a contagem de quadradinhos na malha quadriculada. Essa dificuldade diz respeito à falta de uma habilidade que, segundo consta na BNCC (BRASIL, 2018), deve ser desenvolvida por alunos dos anos iniciais e que, portanto, precisa ser devidamente compreendida por seus professores.

\section{Conclusões}

O objetivo deste trabalho foi analisar as dificuldades de futuros pedagogos no uso de seus conhecimentos para solucionar problemas que envolvem a geometria ensinada nos anos iniciais do Ensino Fundamental. Pelas análises, constatou-se que os participantes tiveram dificuldades para interpretar e solucionar problemas geométricos no que tange às quatro etapas de resolução de problemas propostas por Proença (2018) (representação, planejamento, execução e monitoramento).

$\mathrm{Na}$ etapa da representação do problema, as dificuldades foram relacionadas ao uso de conhecimentos esquemáticos. De modo específico, verificaram-se dificuldades na compreensão do conceito matemático de planificação presente no enunciado de $\mathrm{S} 2$ e também no uso de informações irrelevantes para a solução de $S 1$, S2 e S4. Além disso, notou-se o não reconhecimento da essência do problema (cálculo do perímetro) em S5.

Já na etapa de planejamento, as dificuldades tiveram ligação com o conhecimento estratégico (S1, S3 e S5) e, na etapa de execução, com o conhecimento procedimental (S3 e S4). $\mathrm{Na}$ etapa de monitoramento, verificou-se que, por vezes, os futuros pedagogos apresentaram soluções com uma característica procedimental, ou seja, não se preocuparam com estabelecer relação entre o problema solucionado e o contexto do qual faz parte, algo importante na solução de situações do cotidiano.

Quanto à geometria, nas resoluções analisadas, emergiram dificuldades relacionadas aos aspectos conceituais, mostrando que os futuros pedagogos, por vezes, não conseguiram realizar uma análise dos conceitos geométricos presentes nas situações, conforme apontaram Clements e 
Battista (1992). Além disso, notou-se que as habilidades de orientação espacial, visualização e raciocínio espacial, tidas por Santos e Oliveira (2017) como aspectos centrais do processo de ensino-aprendizagem da geometria, não estão bem desenvolvidos nos participantes.

Diante do exposto, este trabalho contribui para evidenciar as dificuldades e os limites de futuros pedagogos no uso de seus conhecimentos matemáticos na resolução de problemas geométricos destinados a alunos dos anos iniciais do Ensino Fundamental. Vê-se, portanto, a necessidade de realização de novas pesquisas sobre a formação inicial de futuros pedagogos, estudos que busquem analisar o desenvolvimento de habilidades de visualização, de raciocínio e de orientação espacial em meio ao processo de resolução de problemas.

\section{Referências}

ALMEIDA, M. B. A formação inicial de professores no curso de pedagogia: constatações sobre a formação matemática para a docência nas séries iniciais do Ensino Fundamental. Orientadora: Maria das Graças de Lima. Coorientadora: Regina Maria Pavanello. 2009. 177 f. Dissertação (Mestrado em Educação para a Ciência e a Matemática) - Programa de PósGraduação em Educação para a Ciência e a Matemática, Universidade Estadual de Maringá, Maringá, 2009. Disponível em: http://www.pcm.uem.br/dissertacao-tese/64. Acesso em: 29 nov. 2021.

ALVES-MAZZOTTI, A. J.; GEWANDSZNADJER, F. O método nas ciências naturais e sociais: pesquisa quantitativa e qualitativa. 2. ed. São Paulo: Pioneira Thomson Learning, 2002.

BARBOSA, C. P. O pensamento geométrico em movimento: um estudo com professores que lecionam Matemática nos anos iniciais do Ensino Fundamental de uma escola pública de Ouro Preto (MG). Orientadora: Ana Cristina Ferreira. 2011. 186 f. Dissertação (Mestrado em Educação Matemática) - Programa de Mestrado Profissional em Educação Matemática, Universidade Federal de Ouro Preto, Ouro Preto, 2011. Disponível em:

http://www.repositorio.ufop.br/jspui/handle/123456789/3074. Acesso em: 28 nov. 2021.

BOGDAN, R. C.; BIKLEN, S. K. Investigação qualitativa em educação: uma introdução à teoria e aos métodos. Portugal: Porto Editora, 1994.

BRASIL. Ministério da Educação. Base Nacional Comum Curricular. Ensino Médio. 3. ed. Brasília: MEC, 2018.

BRITO, M. R. F. Alguns aspectos teóricos e conceituais da solução de problemas matemáticos. In: BRITO, M. R. F. (org.). Solução de problemas e a matemática escolar. 2. ed. Campinas: Alínea, 2010. p. 13-53.

CLEMENTS, D. H.; BATTISTA, M. T. Geometry and spatial reasoning. In: GROUWS, D. A. (ed.). Handbook of research on mathematics teaching and learning: A project of the National Council of Teachers of Mathematics. New York: Macmillan, 1992. p. 420-464.

DANTE, L. R. Projeto Ápis Matemática: $4^{\circ}$ ano. 3. ed. São Paulo: Editora Ática, 2017.

DOUADY, R.; PERRIN-GLORIAN, M. J. Un processus d'apprentissage du concept d'aire de surface plane. Educational Studies in Mathematics, v. 20, p. 387-424, 1989. DOI: https://doi.org/10.1007/BF00315608. 
ECHEVERRÍA, M. P. P. A solução de problemas em matemática. In: POZO, J. I. (org.). A solução de problemas: aprender a resolver, resolver para aprender. Porto Alegre: ArtMed, 1998. p. 43-65.

FONSECA, M. da C. F. R.; LOPES, M. da P.; BARBOSA, M. das G. G.; GOMES, M. L. M.; DAYRELL, M. M. M. S. S. O ensino de Geometria na Escola Fundamental: três questões para a formação do professor dos ciclos iniciais. 3. ed. Belo Horizonte: Autêntica Editora, 2011.

JONES, K. Issues in the Teaching and Learning of Geometry. In: HAGGARTY, L. (ed.). Aspects of Teaching Secondary Mathematics: perspectives on practice. London: RoutledgeFalmer, 2002, p. 121-139.

KRUTETSKII, V. A. The psychology of mathematical abilities in schoolchildren. Tradução de Joan Teller, do russo para o inglês. Chicago: University of Chicago Press, 1976.

MAIA, E. J; PROENÇA, M. C. de. A resolução de problemas no ensino da geometria: dificuldades e limites de graduandos de um curso de pedagogia. Revista Eletrônica de Educação Matemática, Florianópolis, v. 11, n. 2, p. 402-417, 2016. DOI: https://doi.org/10.5007/19811322.2016v11n2p402.

MARTINS, J. B. J. Relação entre formação docente e desempenho de alunos dos anos iniciais do Ensino Fundamental na resolução de problemas matemáticos. Orientadora: Maria Lídia Sica Szymanski. 2016. 141 f. Dissertação (Mestrado em Educação) - Programa de PósGraduação em Educação, Universidade Estadual do Oeste do Paraná, Cascavel, 2016. Disponível em: http://tede.unioeste.br/handle/tede/3383. Acesso em: 28 nov. 2021.

MAYER, R. E. Thinking, problem solving, cognition. 2. ed. New York: WH Freeman and Company, 1992.

MENEZES, L.; SERRAZINA, L.; FONSECA, L.; RIBEIRO, A.; RODRIGUES, M.; VALE, I.; BARBOSA, A.; CASEIRO, A.; MARTINS, A.; LOUREIRO, C.; FERNANDES, F.; VELOSO, G.; GOMES, H.; BRUNHEIRA, L.; ALMEIDA, P.; TEMPERA, T. Conhecimento de Geometria de estudantes da Licenciatura em Educação Básica. In: SEMINÁRIO DE INVESTIGAÇÃO EM EDUCAÇÃO MATEMÁTICA, 25., 2014, Braga. Anais [...]. Braga: APM, 2014. p. 243-261. Disponível em: http://hdl.handle.net/10400.19/2069. Acesso em: 28 nov. 2021.

PESSOA, G. da S. Um estudo diagnóstico sobre o cálculo da área de figuras planas na malha quadriculada: influência de algumas variáveis. Orientadora: Paula Moreira Baltar Bellemain. 2010. 146 f. Dissertação (Mestrado em Educação Matemática e Tecnológica) Programa de Pós-Graduação em Educação Matemática e Tecnológica, Universidade Federal de Pernambuco, Recife, 2010. Disponível em: https://repositorio.ufpe.br/handle/123456789/3944. Acesso em: 28 nov. 2021.

PROENÇA, M. C. de. Resolução de Problemas: encaminhamentos para o ensino e a aprendizagem de Matemática em sala de aula. Maringá: Eduem, 2018.

RODRIGUES, M. P. P.; SERRAZINA, L. Reconhecimento de figuras no plano a partir da identificação de propriedades. In: ENCONTRO DE INVESTIGAÇÃO EM EDUCAÇÃO MATEMÁTICA, 2017, Lisboa. Anais [...] Lisboa: Universidade de Lisboa, 2017.

ROSA, M. de S. Linguagem Matemática e Resolução de Problemas: Percepção e utilização na formação inicial do Pedagogo. Orientador: Maurício Rosa. 2009. 115 f. Dissertação (Mestrado em Ensino de Ciências e Matemática) - Programa de Pós-Graduação em Ensino de Ciências e Matemática, Universidade Luterana do Brasil, Canoas, 2009. Disponível em: 
http://www.ppgecim.ulbra.br/teses/index.php/ppgecim/article/view/120/113. Acesso em: 28 nov. 2021.

SANTOS, L; OLIVEIRA, H. O ensino e a aprendizagem da geometria: perspectivas curriculares. In: ENCONTRO DE INVESTIGAÇÃO EM EDUCAÇÃO MATEMÁTICA, 2017, Lisboa. Anais [...].

Lisboa: Universidade de Lisboa, 2017.

STERNBERG, R. Psicologia cognitiva. Tradução de Maria Regina Borges Osório. Porto Alegre: ArtMed, 2000, 494p. 